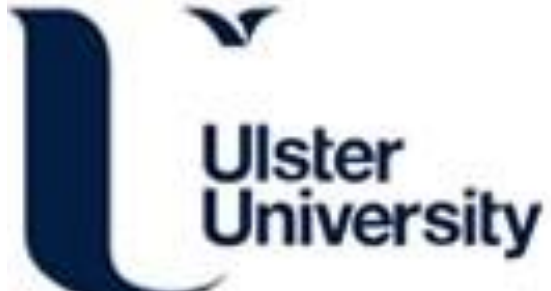

Process support for continuous, distributed, multi-party healthcare processes -
applying workflow modelling to an anticoagulation monitoring protocol

McChesney, I. R. (2016). Process support for continuous, distributed, multi-party healthcare processes applying workflow modelling to an anticoagulation monitoring protocol. In C. R. Garcia, P. Caballero-Gil, M. Burmester, \& A. Quesada-Arencibia (Eds.), Unknown Host Publication (pp. 255-266). Springer. https://doi.org/10.1007/978-3-319-48746-5_26

Link to publication record in Ulster University Research Portal

Published in:

Unknown Host Publication

Publication Status:

Published online: 02/11/2016

DOI:

10.1007/978-3-319-48746-5_26

Document Version

Author Accepted version

\section{General rights}

Copyright for the publications made accessible via Ulster University's Research Portal is retained by the author(s) and / or other copyright owners and it is a condition of accessing these publications that users recognise and abide by the legal requirements associated with these rights.

\section{Take down policy}

The Research Portal is Ulster University's institutional repository that provides access to Ulster's research outputs. Every effort has been made to ensure that content in the Research Portal does not infringe any person's rights, or applicable UK laws. If you discover content in the Research Portal that you believe breaches copyright or violates any law, please contact pure-support@ulster.ac.uk. 


\title{
Process support for continuous, distributed, multi-party healthcare processes - applying workflow modelling to an anticoagulation monitoring protocol
}

\author{
Ian McChesney \\ School of Computing and Mathematics, Ulster University, Newtownabbey, Northern Ireland, \\ UK \\ ir.mcchesney@ulster.ac.uk
}

\begin{abstract}
Workflow management has been shown to be a promising approach to the support of a range of healthcare processes, with tools available for their formal specification, analysis and implementation. To further illustrate its relevance, we apply a workflow modelling approach to the specification and analysis of an anticoagulation monitoring protocol, illustrating a Petri Net-based solution using YAWL and Coloured Petri Nets. The selected scenario is representative of healthcare processes which have not been extensively considered for workflow solutions in the past - namely highly distributed, multi-party activities executing over an extended period of time. In presenting a workflow analysis for such a case, we identify challenges in supporting these types of primary and community care-based processes and identify possible areas in which workflow solutions could be extended to address their particular process requirements.
\end{abstract}

Keywords: Workflow modelling, Petri nets, Healthcare processes, Community care

\section{Introduction}

In this paper we focus on workflow modelling as one approach to designing and enabling healthcare processes. Workflow modelling seeks to understand organizational tasks holistically, viewing workflows as a set of interrelated tasks typically crossing internal and external organizational boundaries to form a complete system of connected activities and people. For this reason, workflow modelling has found relevance in the analysis and support of a range of healthcare processes, for example [1,2,3].

A trend in healthcare over the past say 15 years [4] has been the move towards preventative treatments in primary care and through community care processes - processes which can be understood as continuous, distributed, multi-party workflow systems. In this paper we review workflow modelling approaches for this type of process and describe, as an illustrative example, a simple workflow modelling solution based on Petri Nets for an anticoagulation monitoring protocol. In so doing, we illustrate the value of workflow modelling for process understanding, simulation and implementation, and 
identify some important characteristics of primary and community care processes which merit further investigation for process support.

The remainder of the paper is organized as follows: In Section 2 we review workflow modelling techniques as they have been applied to healthcare processes. Section 3 introduces the case study of an anticoagulation management protocol. Section 4 describes the modelling approach adopted and presents a YAWL (Yet Another Workflow Language) [5] and CPN (Coloured Petri Nets) [6] representation of the case study. Section 5 presents some observations arising from our models and in Section 6 conclusions are drawn.

\section{Related work}

The original application of workflow management techniques to clinical workflow is attributed to Dadam et al [7], who noted the tension between the need for formal modelling of critical processes while allowing for flexibility and ad-hoc variation in their implementation. In a review of work since then, Gooch and Roudsari [3] identify the key challenges in the implementation of information systems to support an "idealized clinical workflow". They emphasize the need to support adaptive care pathways adapting to both clinical and organizational changes. The need for adaptive processes are both an opportunity and a challenge for workflow management solutions.

Workflow modelling and workflow management systems are concerned with the understanding of organizational tasks as a whole to achieve some business or organizational objective. Work Systems Theory [8,9] provides a framework which reinforces the notion of organizational processes as a work system. It makes two important distinctions; first that the customer (or in our case patient) is preeminent in understanding the purpose of any workflow and second, that the development of a workflow system cannot be understood as a one-off processes, but is a system which is in a cycle of initiation, development, implementation and operation \& maintenance.

A full review of workflow modelling approaches as applied in healthcare is beyond the scope of this paper. By way of summary, there are approaches which are concerned with one or a combination of (a) "modelling for understanding", involving construction of models to aid communication between system developer and stakeholders, for example [10,11], (b) "modelling for simulation", seeking to construct a workflow model of the healthcare process with a view to its analysis through visualization and performance analysis, for example [12,13], and (c) "modelling for implementation", concerned with the enablement of a workflow system through a workflow engine, for example $[14,15]$.

\section{Case study overview}

Workflow modelling in healthcare has tended to focus on intra-organization processes, such as acute care in a hospital or processing of patients in an accident and emergency department $[16,17,18]$. In this paper we examine a primary and community care process 
concerned with the ongoing monitoring and management of patients undergoing anticoagulation treatment for either prevention of disease or as therapy for an ongoing condition $[19,20]$.

The case study is representative of those healthcare processes which we describe as continuous, distributed, multi-party processes. Continuous in the sense of executing over an extended period of time, sometimes indefinitely until some significant event occurs. Distributed in the sense that they are located across multiple physical locations, with different activities possibly taking places at different locations (e.g. health clinic, pharmacy, home) and multi-party in that the process is undertaken by a group of participants, typically physically dispersed, whose actions must be coordinated for a successful workflow.

\section{Overview of an anticoagulant monitoring process.}

The process is concerned with patients who are referred from secondary care (hospital) to primary care (their local General Practitioner - GP) for the purpose of anticoagulation treatment and monitoring. This is achieved through GP consultation, patient education, dosage specification and adjustment, regular blood tests for INR (international normalized ratio) measurement (either in clinic or domiciliary) and periodic review.

Referral from secondary care and treatment initiation: Patients are referred to primary care from secondary care using an agreed transfer process. This process includes the transfer of documentation and patient record details for existing and new patients.

Medication and monitoring: On receipt of the prescription by the patient's preferred pharmacy, delivered either in person by the patient or directly from the GP Practice, the pharmacy will dispense the tablets. Along with the dosage instruction, the patient is then able to begin treatment. A characteristic of anticoagulation treatment is regular INR monitoring, typically 8-12 weeks, which may lead to a change in dosage.

Dosage adjustment: The dosage regime (whether changed or unchanged) will be formally notified to the patient. There is a detailed protocol for how this is to be achieved depending on whether the patient is told directly by telephone or through a carer, whether they are living in a care home, and whether a monitored dosage system is in use.

Discontinuation: Any decision to discontinue will be based on a formal consultation with the GP.

\section{$4 \quad$ Modelling Approach}

We follow and extend Jorgensen et al's approach [21] for modelling a healthcare workflow system. This approach is consistent with recommendations [22] to use of a variety of modelling and diagram types when developing healthcare systems - in early stages, choice of model should be determined by the usability of the notation for communication between modeller and stakeholder. We describe and adapt the four stages as below: 


\subsection{Informal Description}

The means through which a healthcare process will initially be described will vary depending on context. In our case, the starting point is a text-based description of the anticoagulation monitoring process setting out tasks, participants and supporting documents.

\subsection{Workflow Requirements Model}

YAWL is both a language and environment for the modelling and implementation of workflow systems. It adopts a state-based modelling approach based on the ideas of Petri Nets and a comprehensive set of workflow patterns. Control flow in a workflow system is modelled using tasks, conditions (implicit or explicit) and flow relations. These are used to form an "extended workflow net"; a set of these nets can be organized hierarchically to form a workflow specification.

Figure 1 shows the top level workflow net for the anticoagulant monitoring case. This uses a subset of the YAWL notation which is fully described in [5]. The focus of the notation is on control flow, in this case from the perspective of the patient as they "flow" or "move" through the system. YAWL also provides support for the modelling of workflow data and resources.

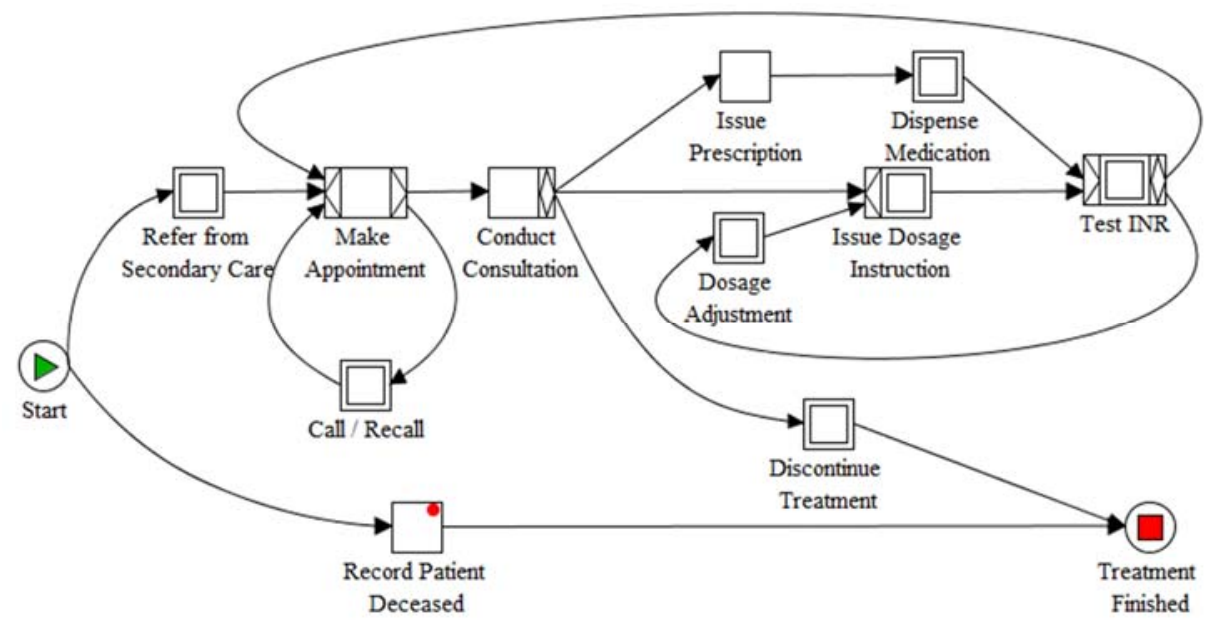

Fig. 1. Top level workflow net for anticoagulant monitoring

The first task in the workflow is Refer from Secondary Care. This is shown as a composite task, indicating that it consists of further detail which is described in a separate extended workflow net which may in turn consist of atomic or composite tasks. Make Appointment is modelled as an atomic task which may take be triggered by three possible inputs: a referral from secondary care, as a result of the call/recall procedure (when a patient does not attend for an appointment), or based on the outcome of an INR test 
result. Since any one appointment will be due to only one of these preconditions, the task is modelled as an XOR-join task. If a patient attends their appointment, the Conduct Consultation task can then take place, otherwise the Call/Recall task is invoked. Make Appointment is modelled as an XOR-split task, ensuring that only one of the two outcomes is possible.

Conduct Consultation is an example or an OR-split task, whereby the thread of control can be passed to one or more of the outgoing arcs. In this case, the possible outcomes are (re)issue of a prescription, set up and issue of dosage instruction or discontinuation of treatment. This is not an XOR condition as the Issue Prescription and Issue Dosage Instruction tasks may both be necessary.

The remainder of the workflow net and notation can be interpreted by the reader. One point to note is the Record Patient Deceased task. YAWL permits the specification of cancellations sets. These are tasks which may be associated with some form of cancellation event which, on execution, causes the set of tasks associated with it to be terminated.

\subsection{Workflow Specification Model}

Coloured Petri Nets is a formal, graphical language for the modelling and analysis of any type of concurrent system. It combines the key concepts of Petri Nets (places/transitions), tokens with data values and the ability to programmatically describe system behaviour and data using the programming language CPN ML [6]. Workflows expressed in YAWL can be easily translated to their CPN equivalent. Further, as a formal representation, CPNs can be executed using environments such as CPN Tools [23], enabling their verification and simulation. In this way, CPNs are suitable for checking workflow requirements, constructing a formal specification of the system and conducting simulation and performance analysis.

We use Coloured Workflow Nets (CWNs) to assemble the specification model for anticoagulant monitoring workflow. CWNs are Coloured Petri Nets with restrictions on their data types to enforce specification of tasks from the perspective of the workflow system [24]. The CWN is a visually more complex representation than the YAWL model. State is explicitly represented and places, transitions and arcs are annotated with rules (expressed in CPN ML) which control the specific behaviour of the system. The following subsections describe aspects of the anticoagulant monitoring CWN in more detail. We assume the reader is familiar with the basic concepts of Petri Nets.

Modelling for understanding - control flow.

Workflow patterns described in YAWL can be mapped directly to a CPN representation. Figure 2 shows the $\mathrm{CWN}$ elements for modelling referral from secondary care. 


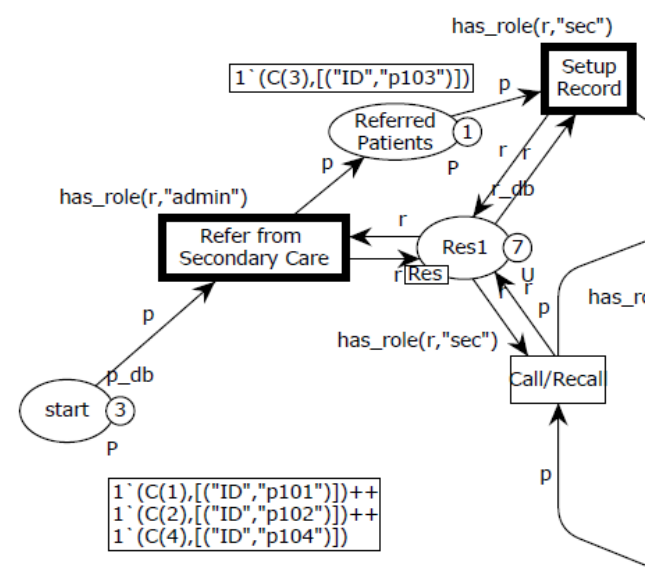

Fig. 2. CWN segment for referral from secondary care. Bold borders show enabled transitions.

This segment models a sequential workflow. Place start represents the point at which referral from secondary care begins. In this example, there are patients awaiting referral (p101, p102, p104). The firing of the Refer from Secondary Care transition represents a patient referral being handled. This transition uses a hospital admin resource, as specified by the has_role function on the transition. When the task is complete, the referred patient $p$ is moved through the system, as represented by the place Referred Patients. Also on completion of the Refer from Secondary Care transition, the admin resource is released back to the resource pool - modelled as a CPN fusion place.

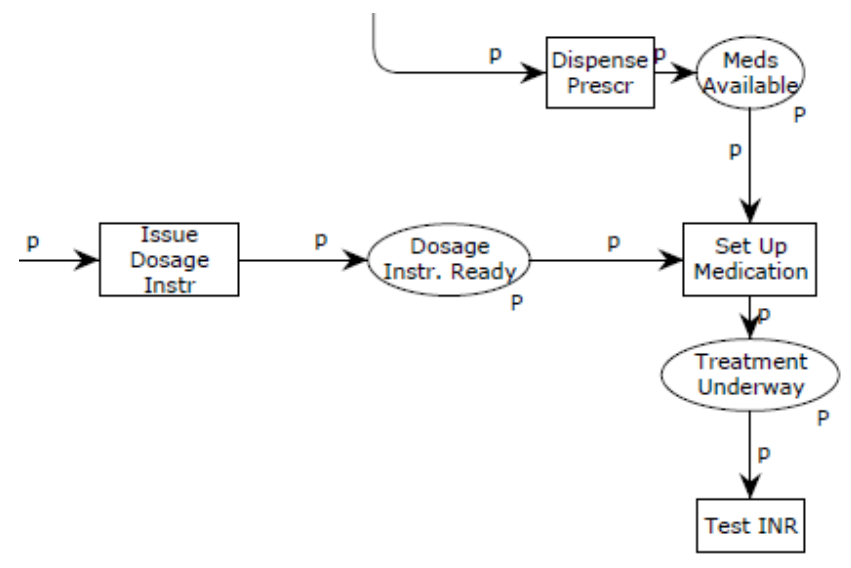

Fig. 3. CPN representation of an AND-join - Test INR

As an example of synchronization, the AND-join Test INR (from Figure 1) is represented in CPN as shown in Figure 3 (resources are omitted for readability). In Figure 4 we can see a case of simple merge where the YAWL XOR-join for Make Appt is modelled. There are three possible workflow sequences which can require an appointment to be made: referral from secondary care (transition Setup Record), a requirement for a 
review appointment (transition Set up Review), or if a patient is being identified through the call/recall procedure (transition Call/Recall). The CWN is showing that the Make Appt task requires action (for p103) and the Call/Recall task requires action (for p102). There is a CPN ML function attends associated with the Make Appt transition, modelling whether a patient will attend their appointment:

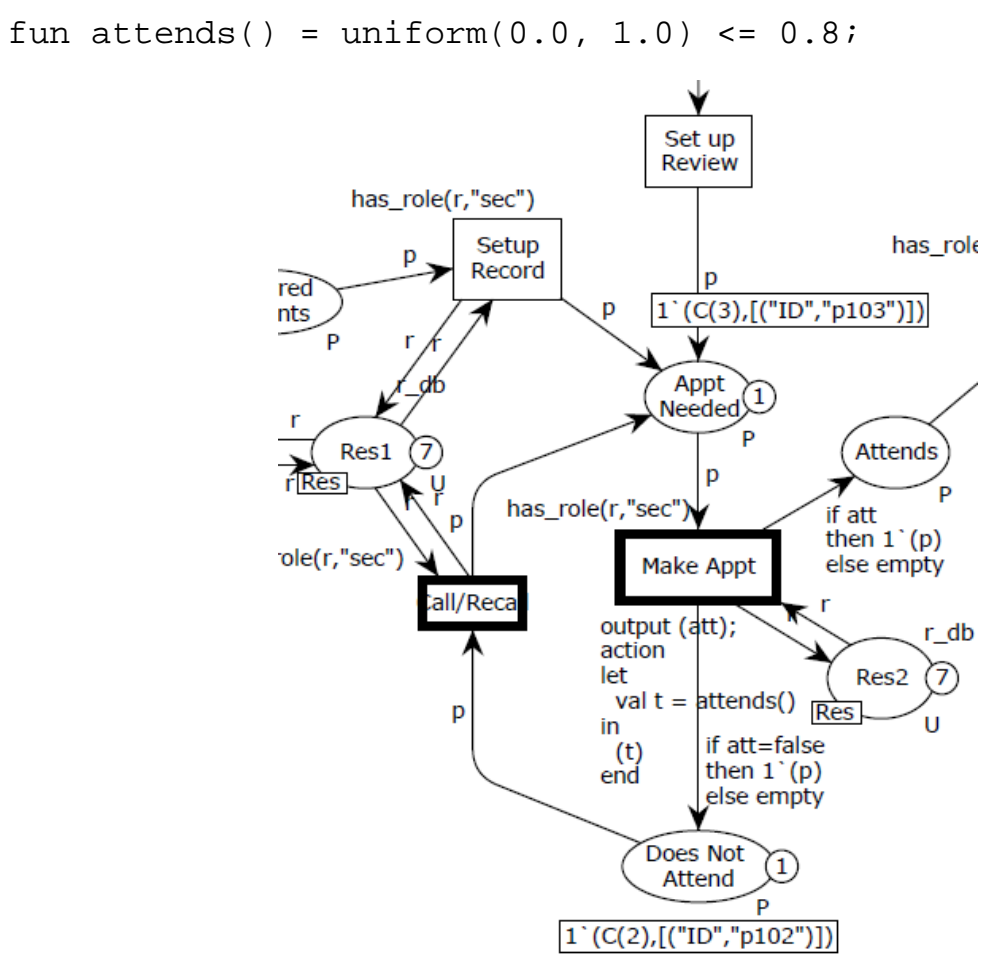

Fig. 4. CPN representation of an XOR-join - Make Appt

\section{Modelling for simulation.}

Having established that the CWN correctly reflects the required workflow functionality, it can be extended with timing properties to enable simulation and performance analysis. This can help answer questions such as: how many INR tests are likely to be conducted per month? How many review appointments can be expected per month? How much time do we expect to spend on call/recall appointments?

CPN ML functions can be used to simulate workflow behaviour. The attends function (Figure 4) has already been highlighted to model the frequency with which appointments are kept. The probability distribution can be changed to reflect different simulation scenarios. Additional functions are used in this model to simulate the need for patient review and discontinuation of treatment. The CWN can also simulate the referral of new patients from secondary care into the system (see e.g. [25] for approach).

To illustrate the possibilities through simulation, we consider a version of the CWN which simulates the handling of 80 referrals over a period of time. We assume that a 
patient is referred randomly every 14-28 days. We make other assumptions for simulation purposes regarding how long certain tasks take to complete, for example, 2 days to complete the referral paperwork, 1 day to set up an appointment, 2 days to generate a repeat prescription. From this position, we can establish some performance statistics through simulation such as :

$\begin{array}{ll}\text { Observation } & \text { Count } \\ \text { Number Of Patient Reviews } & 669 \\ \text { Instances of SetUpMedication } & 4290 \\ \text { Model time (days) } & 3666\end{array}$

Model time is in days, so 3666 days represents 131 months (effectively an 11 year cycle for the throughput of 80 patients undergoing anticoagulation treatment). Under these assumptions we have on average 5.12 patient reviews per month and 32.75 medication setups per month. We can similarly simulate a more steady state scenario where say 80 patients are "in the system" and then simulate system performance during a period when a further 10 referrals are received.

\subsection{Workflow Implementation with YAWL}

The purpose of the CPN representation is to confirm a correct understanding of the system, to enable simulation, and to allow for a range of different types of performance analysis. CPN is not suitable for implementation or enablement as a live workflow system. There are numerous options for how this might be achieved. Here we illustrate how the YAWL system facilitates this.

In the same way that a CPN version of an existing YAWL workflow net can be easily constructed, similarly the YAWL equivalent of the CPN specification model can be constructed. With the CWN representation having articulated the resource requirements of the workflow, these resources can also be mapped directly into the YAWL Environment.

The YAWL system enables execution of a workflow net as a workflow case. In addition to the control-flow aspect of the workflow, as specified visually in the YAWL Editor, the YAWL environment permits the specification of data associated with the workflow and resources to enact the workflow. For example, referral of a patient from secondary care is achieved via a Referral Form (this is identified in the sub-net associated with referral from secondary care). In YAWL, this can be specified as a data object associated with the Refer From Secondary Care task using the built-in complex datatype YDocumentType.

Organizational structures can be specified in the YAWL system terms of roles, capabilities, positions and organization groups. For each role, individual users of the workflow system can be specified, along with their privileges and their place within the organizational structure (Figure 5).

When a case is executing, the YAWL Environment, using the workflow specification, manages the execution flow in terms of, for example, work items currently awaiting execution. Depending on their configuration, work items can be allocated to a resource manually or automatically. The significance to note is that, having translated the 
CWN into YAWL, the workflow execution is based on a formally verified CPN model of the workflow requirements.

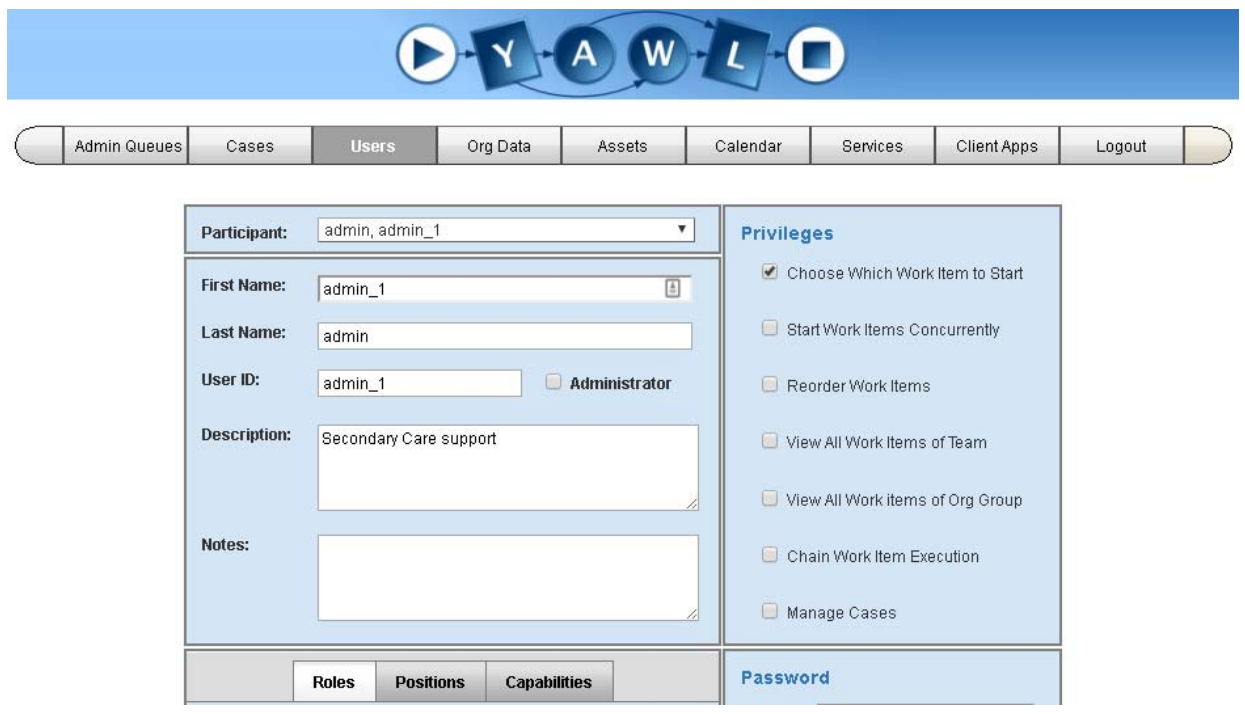

Fig. 5. Setting up users in the workflow system

\section{Observations}

As noted above, we have selected our case study as representative of healthcare processes which are continuous, distributed and multi-party. We present a number of observations regarding the modelling of such processes. Strengths of the workflow approach for this type of scenario include:

- the workflow model is patient centered. Process and state is modelled from the perspective of the patient as they "move" through the system. This supports the notions of Work Systems Theory in which the "customer" is central to the process. The model seeks to ensure the patient does not "get lost" in the system, that every action performed on a patient case has achievable preconditions and postconditions.

- our approach begins and ends with YAWL. This addresses the need for modelling which can be used in stakeholder communication and the need for a model which is executable. This also supports the notion of a workflow system as being in a cycle of evolution rather than a one-off project - the notation used for "modelling for understanding" is also the notation for implementation, supporting cyclical evaluation and improvement.

- workflow exceptions can be modelled through the suite of workflow patterns supported by YAWL and CPN. Functional accuracy and correctness can be verified in the CPN model before translation to YAWL for implementation.

- though the example above presents a flat CPN, a hierarchical model can be developed reflecting the hierarchy in the domain and the original YAWL specification. 
This enables a loose coupling of different workflow components, facilitating the management of complexity.

- an executable workflow model enables process awareness. For example, the YAWL workflow engine ensures that each participants' obligations and next steps can be explicitly modelled and notified to them.

- in simulation mode (CWN) or execution mode (YAWL system), the model can generate event logs of actual process. This enables the application of process mining for process enforcement and process enhancement [26].

Characteristics of this type of process which present challenges from a modelling perspective include the following:

- in such multi-party and distributed processes, message exchange is a key coordination mechanism. When an unexpected event occurs (for example, the pharmacy will not have the required medication for 24 hours), how do we model who should be informed?

- how can the correct level of support be provided to participants who are typically working in different organizations or healthcare teams? In establishing the desired level of coordination, there are many choices to be made in terms of control and flexibility. We note Cabitza and Simone's alpha-level taxonomy as providing a useful framework for conceptualizing this problem [27].

- resources modelled in CWNs and YAWL are static, yet real-world processes typically have different participants moving in and out of the workflow (for example, personnel in care support teams, locum GPs). How is resource allocation to be managed to ensure continuity of the workflow and continuity of care?

- there is no explicit workflow concept of location awareness. For example, can workflow efficiencies be achieved where multiple executing instances identify similar tasks in the same geographical areas such as domiciliary INR testing or replenishment of supplies?

- how are the coordination mechanisms which are necessary between participants to be supported? For example, can process awareness best be supported in a distributed fashion or centrally though control from the workflow owner, for example GP surgery.

\section{Conclusions}

Modelling, analyzing and implementing a continuous, distributed, multi-party healthcare processes with complex coordination requirements has been presented through an approach using established Petri Net and workflow technologies. We have argued that such a workflow approach is patient centric, and addresses the requirement of a mixed modelling notation which supports a process cycle of ongoing improvement.

By illustrating how YAWL and CPN can be used for this, we have highlighted process characteristics which can be modelled in this way, be it modelling for understanding, for analysis or for implementation. Further work will require refinement of model 
parameters to reflect different healthcare process scenarios for realistic performance modelling purposes.

Primary care, community care and home care processes present many challenges in ensuring their efficient operation. We have shown how existing tools for workflow modelling and management are sufficiently expressive to capture much of these. However, there are many technical opportunities for further work, for example [28,29], to deliver process enhancement for this class of healthcare process.

\section{REFERENCES}

1. Schadow, G., Russler, D.C. and McDonald, C.J., Conceptual alignment of electronic health record data with guideline and workflow knowledge. International journal of medical informatics, 64(2), pp.259-274. (2001)

2. Fox, J., Black, E., Chronakis, I, Dunlop, R, Patkar, V., South, M. and Thomson, R. From guidelines to careflows: modelling and supporting complex clinical processes. In: ten Teije, A, Miksch, S and Lucas, P. (eds) Computer-based medical guidelines and protocols: a primer and current trends, 139, pp.44-62. IOS Press, Amsterdam (2008)

3. Gooch, P. and Roudsari, A., Computerization of workflows, guidelines, and care pathways: a review of implementation challenges for process-oriented health information systems. Journal of the American Medical Informatics Association, 18(6), pp.738-748. (2011)

4. Genet, N., Boerma, W., Kroneman, M., Hutchinson, A. and Saltman, R.B. (eds) Home care across Europe - Current structure and future challenges. London: European Observatory on Health Systems and Policies. (2013)

5. van der Aalst, W.M. and Ter Hofstede, A.H., YAWL: yet another workflow language. Information systems, 30(4), pp.245-275. (2005)

6. Jensen, K., Kristensen, L.M. and Wells, L., Coloured Petri Nets and CPN Tools for modelling and validation of concurrent systems. International Journal on Software Tools for Technology Transfer, 9(3-4), pp.213-254. (2007)

7. Dadam, P., Reichert, M. and Kuhn, K.. Clinical Workflows - the killer application for process-oriented information systems?. In BIS 2000, 4th International Conference on Business Information Systems, Poznan, Poland, 12-13 April 2000 (pp. 36-59). Springer London. (2000)

8. Alter, S. Using work system theory to link managerial and technical perspectives on BPM. In: 2013 IEEE International Conference on Business Informatics, pp. 222-227. IEEE Press, New York (2013)

9. Alter, S. Work system theory: overview of core concepts, extensions, and challenges for the future. Journal of the Association for Information Systems, 14(2), p.72. (2013)

10. Fanti, M.P., Mininel, S., Ukovich, W. and Vatta, F. Modelling alarm management workflow in healthcare according to IHE framework by coloured Petri Nets. Engineering Applications of Artificial Intelligence, 25(4), pp.728-733. (2012)

11. Ruiz, F., Garcia, F., Calahorra, L., Llorente, C., Gonçalves, L., Daniel, C. and Blobel, B.. Business process modeling in healthcare. Studies in Health Technology and Informatics, 179, pp.75-87. (2012)

12. Jansen-Vullers, M.H. and Reijers, H.A. Business process redesign at a mental healthcare institute: A coloured Petri net approach. In: Proceedings of the Sixth Workshop and Tutorial on Practical Use of Coloured Petri Nets and the CPN Tools (PB-576) pp. 21-38. (2005)

13. Elnahrawy, E. and Martin, R.P. Studying the utility of tracking systems in improving healthcare workflow. In: 8th IEEE International Conference on Pervasive Computing and 
Communications Workshops (PERCOM Workshops), pp. 310-315. IEEE Press, New York (2010)

14. van der Aalst, W.M., Aldred, L., Dumas, M. and ter Hofstede, A.H. Design and implementation of the YAWL system. In: International Conference on Advanced Information Systems Engineering, pp. 142-159. Springer, Heidelberg. (2004)

15. Mans, R., van der Aalst, W., Russell, N., Moleman, A., Bakker, P., \& Jaspers, M. YAWL4Healthcare. In: Hofstede, A.H., van der Aalst, W.M., Adams, M. and Russell, N. (eds) Modern Business Process Automation, pp. 543-565. Springer, Heidelberg. (2010)

16. Vanberkel, P.T., Boucherie, R.J., Hans, E.W., Hurink, J.L. and Litvak, N. A survey of health care models that encompass multiple departments. University of Twente, Enschede, http://www.math.utwente.nl/publications/ (2009)

17. Rohleder, T.R., Lewkonia, P., Bischak, D.P., Duffy, P. and Hendijani, R. Using simulation modeling to improve patient flow at an outpatient orthopedic clinic. Health care management science, 14(2), pp.135-145 (2011)

18. Barrios, M.A.O., Caballero, J.E. and Sánchez, F.S. A Methodology for the Creation of Integrated Service Networks in Outpatient Internal Medicine. In: Bravo, J., Hervás, R. and Villarreal, V. (eds) Ambient Intelligence for Health, Proc. 1st International Conference, AmIHEALTH. LNCS, vol. 9456 pp. 247-257. Springer, Heidelberg (2015)

19. Blann, A.D., Fitzmaurice, D.A. and Lip, G.Y. Anticoagulation in hospitals and general practice. British medical journal, 326(7381), p.153. (2003)

20. Keeling, D., Baglin, T., Tait, C., Watson, H., Perry, D., Baglin, C., Kitchen, S. and Makris, M., Guidelines on oral anticoagulation with warfarin-fourth edition. British journal of haematology, 154(3), pp.311-324. (2011)

21. Jørgensen, J.B., Lassen, K.B. and van der Aalst, W.M. From task descriptions via colored petri nets towards an implementation of a new electronic patient record workflow system. International Journal on Software Tools for Technology Transfer, 10(1), pp.15-28. (2008)

22. Jun, G.T., Ward, J., Morris, Z. and Clarkson, J. Health care process modelling: which method when?. International Journal for Quality in Health Care, 21(3), pp.214-224 (2009)

23. CPN Tools, http://cpntools.org/

24. van der Aalst, W.M., Jørgensen, J.B. and Lassen, K.B. Let's go all the way: From requirements via colored workflow nets to a BPEL implementation of a new bank system. In: Meersman, R. and Tari, Z (eds.) OTM Confederated International Conferences, "On the Move to Meaningful Internet Systems" (pp. 22-39). Springer, Heidelberg (2005)

25. Jensen, K. and Kristensen, L.M. Coloured Petri nets: modelling and validation of concurrent systems. Springer, Heidelberg (2009)

26. van der Aalst, W,M., Process Mining: Discovery, Conformance and Enhancement of Business Processes. Springer, Heidelberg (2011)

27. Cabitza, F. and Simone, C. Computational Coordination Mechanisms: A tale of a struggle for flexibility. Computer Supported Cooperative Work, 22(4-6), pp.475-529. (2013)

28. Toutain, F., Le Huérou, E. and Beaufils, E. On webco interoperability. In: Proceedings of the 1st Workshop on All-Web Real-Time Systems Article 5, 6 pages ACM, NY. (2015)

29. Tolkiehn, G., Lebedev, M. and Makariti, A. MyWebRTC, a free do-it-yourself kit for secure real-time internet-communication. In Information Technologies in Innovation Business Conference (ITIB), 2015 pp. 81-84. IEEE Press, NY (2015) 